\title{
Downregulation of the long non-coding RNA MEG3 promotes osteogenic differentiation of BMSCs and bone repairing by activating Wnt/ $\beta$-catenin signaling pathway
}

Juan Liu

Shanghai First People's Hospital: Shanghai Jiaotong University First People's Hospital

Xin Qi ( $\nabla$ qixin19871012@163.com )

Shanghai Pudong Hospital

Hong-Sheng Miao

Shanghai First People's Hospital: Shanghai Jiaotong University First People's Hospital

Zi-Chao Xue

Qingdao Municipal Hospital Group

\section{San-Hu Zhao}

Shanghai First People's Hospital: Shanghai Jiaotong University First People's Hospital

Guo-Yi Gao

Shanghai First People's Hospital: Shanghai Jiaotong University First People's Hospital

Mei-Qing Lou

Shanghai First People's Hospital: Shanghai Jiaotong University First People's Hospital

Cheng-Qing Yi

Shanghai Pudong Hospital

\section{Research Article}

Keywords: long non-coding RNA, MEG3, BMSCs, skull defect, bone regeneration

Posted Date: April 19th, 2021

DOI: https://doi.org/10.21203/rs.3.rs-413260/v1

License: (9) This work is licensed under a Creative Commons Attribution 4.0 International License.

Read Full License 


\section{Abstract}

Background: Previous studies have demonstrated long non-coding RNA maternally expressed gene 3 (MEG3) emerged as a key regulator in development and tumorigenesis. However, whether MEG3 participate in osteogenic differentiation and bone regeneration remains unclear. This study aims to investigate the function and mechanism of MEG3 in osteogenic differentiation of bone marrow mesenchymal stem cells (BMSCs), and explores the use of MEG3 in skull defects bone repairing.

Methods: Endogenous expression of MEG3 during BMSCs osteogenic differentiation were detected by qPCR. MEG3 was knockdown in BMSCs by lentivirus. The proliferation, osteogenic-related genes and proteins expression were assessed by the CCK-8, PCR, alizarin red and alkaline phosphatase staining in MEG3 knockdown BMSCs. Western blot was used to detected $\beta$-catenin expression in MEG3 knockdown BMSCs. DKK1 was used to block wnt/ $\beta$-catenin pathway, the osteogenic-related genes and proteins expression were assessed by PCR, alizarin red and alkaline phosphatase staining in MEG3 knockdown BMSCs. MEG3 knockdown BMSCs scaffold with PHMG were implanted in a critical-sized skull defects of rat model, micro-CT, hematoxylin and eosin staining, and immunohistochemistry were performed to evaluate the bone repairing.

Results: MEG3 was increased during osteogenic differentiation of BMSCs. Downregulation of MEG3 could promote osteogenic differentiation of BMSCs in vitro. Notably, a further mechanism study revealed MEG3 knockdown could activate Wnt/ $\beta$-catenin signaling pathway in BMSCs. Wnt/ $\beta$-catenin inhibition would impair MEG3-induced osteogenic differentiation of BMSCs. By using PHMG scaffold with MEG3 knockdown BMSCs, we found that downregulation of MEG3 in BMSCs could accelerated bone repairing in a critical-sized skull defects rat model.

Conclusions: Our study reveals the important role of MEG3 during osteogenic differentiation and bone regeneration. Thus, MEG3 engineered BMSCs may be effective potential therapeutic targets for skull defects.

\section{Background}

In the clinic, skull defects caused by trauma, severe infection, tumor resection, and decompressive craniectomy due to refractory high intracranial pressure are very common in neurosurgery. Although autologous bone transplantation, allogeneic bone transplantation, titanium mesh or polyether-etherketone (PEEK) cranioplasty were widely used in the clinical treatment of skull defects, complications such as donor site morbidity, bone resorption/loosening graft infection, implant exposure and high costs remain unresolved(1-3). Bone marrow mesenchymal stem cells (BMSCs) retain their self-renewal capability and have the potential to differentiate into a variety of cell types; hence, they are widely used for tissue repairing. Multiple studies have demonstrated combinations of biomaterials, growth factors or gene modified BMSCs-based tissue engineering are promising alternative approach to facilitate bone regeneration, and have acquired satisfied results in repairing critical-sized bone defects(4-6). 
Maternally expressed gene 3 (MEG3) is a maternally expressed long non-coding RNA, and increasing evidences have revealed that MEG3 emerged as a key regulator in the process of development and tumorigenesis $(7,8)$. We have previously reported that downregulation of MEG3 could promotes angiogenesis after ischemic brain injury(9). However, the function of MEG3 in osteogenic differentiation of MSCs and bone regeneration remains largely unknown. Recent studies have revealed MEG3 inhibits the osteogenic differentiation of periodontal ligament cells(10), human dental pulp stem cells(11) and BMSCs from postmenopausal osteoporosis(12). Down-regulated MEG3 promotes osteogenic differentiation of human dental follicle stem cells(13). While Zheng et al. has proved that down-regulation of MEG3 suppresses osteogenic differentiation of human adipose-derived stem cells(14). Upregulation of MEG3 promotes osteogenic differentiation of MSCs from multiple myeloma patients(15). In addition, Liu et al. has showed that MEG3 is up-regulated in non-union bone fracture, and silencing MEG3 could accelerate tibia fraction healing in mice(16). These contradictory findings indicate that dysregulation of MEG3 might play an important role in osteogenic differentiation of MSCs and bone remodeling.

Wnt/ $\beta$-catenin signaling is a crucial pathway in the skeleton controlling osteoblast differentiation and bone formation(17). When wnt/ $\beta$-catenin pathway acting, wnt ligands bind to Frizzled and LRP5/ 6 receptors and induce stabilization of cytoplasmic $\beta$-catenin by inhibiting GSK3 $\beta$. $\beta$-catenin migrates into nuclei and forms a complex with transcriptional factor Tcf/Lef that transactivates target genes of the Wnt signaling pathway $(18,19)$. Previously studies have showed disruption of $\beta$-catenin affects the osteogenic differentiation of BMSCs(20), and leads to extensive bone marrow adiposity and low bone mass(21). Interestingly, crosstalk between MEG3 and Wnt/ $\beta$-catenin pathways affected tumorigenesis(22, 23), osteogenic differentiation(13, 24), and bone formation(16), suggesting MEG3 is an regulator of Wnt signaling.

In this study, we found the expression of MEG3 increased during the process of osteogenic differentiation. Base on previous studies and our results, we hypothesize that MEG3 may participate in osteogenic differentiation of BMSCs and bone regeneration. The function of MEG3 during osteogenic differentiation of BMSCs was demonstrated by assessing the expression levels of osteogenic genes, alkaline phosphatase activity and calcium deposition. Notably, a further mechanism study revealed that the pro-osteogenic effects of MEG3 may be partially attributed to wnt/ $\beta$-catenin signaling pathway. A composite poly (3-hydroxybutyrate-co-3-hydroxyhexanoate, $\mathrm{PHBHHx}$ )-mesoporous bioactive glass (MBG) scaffold (PHMG), composed of biodegradable PHBHHx and MBG, was selected as the vehicle in this study. Owing to the advantages of its good biocompatibility, favorable loading behavior of its mesoporous structure. Our previous studies have shown that composite PHMG scaffolds has no cytotoxicity and improved cellular affinity, furthermore, PHMG possesses hierarchical mesoporous structure with a comparatively large specific surface area for cellular adhesion and spread(5). PHMG scaffold adherent with MEG3 modified BMSCs were implanted in a critical-sized skull defects of rat model for 8 weeks. Bone regeneration was determined using micro-CT, and histologic analyses. Our results show downregulation of MEG3 could enhanced osteogenic differentiation of BMSCs and promoting bone repairing via $\mathrm{Wnt} / \beta$-catenin signaling. 


\section{Materials And Methods}

\section{Cells and reagents}

human bone marrow stromal cells (hBMSCs) were obtained from four donors who gave their written informed consent. Briefly, marrow was extracted from the femoral midshaft and then suspended in minimum essential medium containing 10\% fetal bovine serum (Hyclone; GE Healthcare, Little Chalfont, $\mathrm{UK}), 100 \mathrm{U} / \mathrm{mL}$ penicillin and $100 \mathrm{mg} / \mathrm{L}$ streptomycin. Subsequently, the non-adherent cells were discarded; the adherent cells converged to $80-90 \%$ confluence and were then replated as passage one (P1) cells. P3 cells were used for experiments. A density of $1 \times 10^{5}$ cells $/ \mathrm{mL}$ was used in the cellular tests. Recombinant DKK1 was purchased from PeproTech (Rocky Hill, NJ, USA). In accordance with a previous study, the applied concentration of DKK1 was $0.5 \mu \mathrm{g} / \mathrm{mL}(20,25)$.

\section{Lentiviral packaging and cell infection}

Lentivirus knockdown MEG3 particles and lentiviral RFP particles were described as previously(9). The lentiviral RFP particles were used as control group in this study. For infections, hBMSCs were incubated with lentiviral particles and polybrene $(5 \mu \mathrm{g} / \mathrm{mL})$ in growth medium. After $6 \mathrm{~h}$, the infection medium was discarded. After 3 days, the cells were screened using puromycin $(4 \mu \mathrm{g} / \mathrm{mL}$; Sigma, Shanghai, China) and then passaged for use in subsequent experiments. The expression of MEG3 was quantified by quantitative real-time polymerase chain reaction (qPCR) and immunofluorescence.

\section{Cell Counting Kit-8 (CCK-8)}

To assess the effect of MEG3 downregulation on the proliferation of hBMSCs, the cells were seeded into a 96-well plate (5000/well) and allowed to adhere for $24 \mathrm{~h}$. After $24 \mathrm{~h}$, the medium was removed, and the cells were treated with 10\% CCK-8 (Dojindo, Kumamoto, Japan) in $100 \mu \mathrm{L}$ low-sugar Dulbecco's modified Eagle's medium (L-DMEM) without fetal bovine serum (FBS) for $2 \mathrm{~h}$ at $37^{\circ} \mathrm{C}$. Absorbance at $450 \mathrm{~nm}$, which is directly proportional to cell proliferation, was measured using a microplate reader (ELX808; BioTek, Winooski, VT, USA).

\section{Osteogenic differentiation protocol}

BMSCs were cultured in growth medium [L-DMEM; 10\% FBS (1495527; Gibco, Waltham, MA, USA) and $100 \mathrm{lU} / \mathrm{mL}$ penicillin/streptomycin] in 6- or 12-well cell culture plates (Corning, Shanghai, China), at a density of $3 \times 10^{4} / \mathrm{cm}^{2}$, and incubated for $48 \mathrm{~h}$ at $37^{\circ} \mathrm{C}$ under $5 \% \mathrm{CO}^{2}$. The cells were subsequently cultured in osteogenic induction medium (L-DMEM with 10\% FBS, $100 \mathrm{lU} / \mathrm{mL}$ penicillin/streptomycin, 100 $\mathrm{nM}$ dexamethasone, $0.2 \mathrm{mM}$ ascorbic acid, and $10 \mathrm{mM} \beta$-glycerophosphate). The cells were maintained by the addition of fresh osteogenic induction medium every 3 days.

\section{Measurement of alkaline phosphatase (ALP) activity}


For the measurement of ALP activity, cells were lysed in radioimmunoprecipitation assay (RIPA) lysis buffer (Beyotime, Shanghai, China), and the lysate $(10 \mu \mathrm{L})$ was incubated with $90 \mu \mathrm{L}$ fresh solution containing p-nitrophenyl phosphate substrate at $37^{\circ} \mathrm{C}$ for $30 \mathrm{~min}$. The reaction was stopped by the addition of $0.5 \mathrm{~N} \mathrm{NaOH}(100 \mu \mathrm{L})$, and the absorbance was measured at $405 \mathrm{~nm}$ using a microplate reader (ELX808; BioTek). The total protein concentration was measured using a BCA protein assay kit (KeyGen BioTECH, Nanjing, China). The relative ALP activity is expressed as the percentage change in optical density $(O D)$ per unit time per milligram protein: $(O D / 15 \mathrm{~min} / \mathrm{mg}$ protein $) \times 100$.

\section{Alizarin red staining (ARS)}

After the induction of osteogenic differentiation, mineral deposition was assessed by ARS (Cyagen Biosciences). Cells were fixed in $4 \%$ paraformaldehyde (Sangon Biotech, Shanghai, China) for 15 min at room temperature and then washed with distilled water. A $1 \%$ solution of alizarin red was added and incubated for $30 \mathrm{~min}$ at room temperature, followed by rinsing with distilled water. The solution was collected, and $200 \mu \mathrm{L}$ were plated on 96 -well plates, which were read at $560 \mathrm{~nm}$ using a microplate reader (ELX808; BioTek). The readings were normalized to the total protein concentration.

\section{RNA isolation and qPCR}

Total cellular RNA was isolated using RNAiso reagent (Takara, Dalian, China) and quantified by measuring the absorbance at $260 \mathrm{~nm}$ (NanoDrop 2000; Thermo Fisher Scientific, Waltham, MA, USA). Total RNA ( $\leq 1000 \mathrm{ng}$ ) was reverse-transcribed into cDNA in a reaction volume of $20 \mu \mathrm{L}$ using the Double-Strand cDNA Synthesis Kit (Takara, Dalian, China). One microliter of cDNA was used as the template for the qPCR reaction. All gene transcripts were quantified by qPCR using the Power SYBR Green PCR Master Mix (Takara) on the ABI StepOnePlus System (Applied Biosystems, Warrington, UK). The mRNAs of the target genes and GAPDH were quantified in separate tubes. All primers were synthesized by Sangon Biotech (Shanghai, China). The primer sequences used are shown in Table 1. The cycle conditions were as follows: $95^{\circ} \mathrm{C}$ for $30 \mathrm{~s}$ and then 40 cycles of $95^{\circ} \mathrm{C}$ for $5 \mathrm{~s}$ and $60{ }^{\circ} \mathrm{C}$ for $30 \mathrm{~s}$. The relative target gene expression levels were calculated using the $2^{-\triangle \Delta \mathrm{Ct}}$ method.

\section{Western blot analysis}

Cells were lysed in RIPA lysis buffer supplemented with a proteasome inhibitor (Beyotime). Total proteins were separated by $10 \%$ sodium dodecyl sulfate polyacrylamide gel electrophoresis and then transferred to a polyvinylidene fluoride membrane (Millipore, Shanghai, China). After blocking in $5 \%$ non-fat milk for 2 $h$, the membranes were incubated overnight at $4{ }^{\circ} \mathrm{C}$ with antibodies specific to $\beta$-actin $(1: 1000, A b c a m$ Inc., USA), or $\beta$-catenin (1:1000, Cell Signaling Technology, USA). Horseradish peroxidase (HRP)conjugated goat anti-rabbit IgG (1:1500; Cell Signaling Technology, USA) was applied as a secondary antibody for $2 \mathrm{~h}$ at room temperature. The immunoreactive bands were detected using an enhanced chemiluminescent detection reagent (Millipore, Shanghai, China). Signal intensity was measured using a Bio-Rad XRS chemiluminescence detection system (Bio-Rad, Hercules, CA, USA). 


\section{Cell seeding}

Prior to seeding cells, the prefabricated PHMG scaffolds were sterilized using gamma irradiation. Cell suspension $(100 \mu \mathrm{L})$ was added to four groups at a density of $1 \times 10^{4}$ cells/scaffold. After $4 \mathrm{~h}, 100 \mu \mathrm{L}$ of culture medium was carefully added to the base of the culture plate until the scaffold was covered with sufficient culture medium.

\section{In vivo evaluation in animals}

Animal experiments were approved by the Research Ethics Committee of the Shanghai General Hospital, and performed in accordance with the Care and Use of Laboratory Animals protocols. Briefly, mature Sprague Dawley (SD) male rats (mean body weight 250-300 g) were provided with sterilized food and water and housed in a barrier facility with a 12-h light/dark cycle. These rats were randomly divided into three groups, each containing six rats: PHMG, PHMG + sh-Ctrl, and PHMG + sh-MEG3 group. For the surgical procedure, as previously described, the animals were anesthetized by intraperitoneal injection of chloral hydrate ( $4 \% ; 9 \mathrm{~mL} / \mathrm{kg}$ body weight) and all operations were performed under sterile conditions. A $1.5-\mathrm{cm}$ sagittal incision was made in the scalp and the calvarium was exposed by blunt dissection. Two critical-sized calvarial defects with a bilateral diameter of $5 \mathrm{~mm}$ were created using a dental trephine, and the scaffolds were then implanted into the defects. Following the operation, the animals received intramuscular antibiotic injections, were allowed free access to food and water and were monitored daily for potential complications. Eight weeks after the operation, the rats were killed by an overdose of anesthetics and their craniums were harvested and fixed in a $4 \%$ paraformaldehyde solution buffered with $0.1 \mathrm{M}$ phosphate solution ( $\mathrm{pH} 7.2$ ) overnight before further analysis.

\section{Micro-computed tomography (CT) evaluation}

All the harvested specimens were examined using the mCT-80 system to evaluate new bone formation within the defect region. Briefly, the undecalcified samples were scanned at a resolution of $18 \mu \mathrm{m}$ and decalcified samples perfused with Microfil ${ }^{\circledR}$ were scanned at a resolution of $9 \mu \mathrm{m}$. After 3D reconstruction, the bone mineral density (BMD) and bone volume fraction (bone volume/total volume [BV/TV]) in the defect regions were used to calculate new bone formation using the auxiliary software of the mCT-80 system.

\section{Histological and Immunohistochemical (IHC) analysis}

The one part of calvarias were decalcified in 10\% EDTA for 14 days, dehydrated with graded ethanol solutions, embedded in paraffin and sectioned at $5 \mu \mathrm{m}$ at the central area of the defect. Sections were stained with hematoxylin and eosin (HE) to observe new bone formation.

The other part of each cranium was decalcified for approximately 2 weeks, dehydrated using a graded alcohol series, embedded in paraffin and sectioned into $5 \mu \mathrm{m}$ sections. Osteocalcin (OCN) IHC was performed to evaluate osteogenesis in specimens. 


\section{Statistical analysis}

Statistical analysis was performed using SPSS 17.0 software (IBM, Armonk, NY, USA). All experiments were performed at least in triplicate, and the data are presented as means \pm standard deviation.

Statistical significance was determined using a two-tailed Student's t-test when comparing two groups, and one-way ANOVA followed by Bonferroni's post hoc test when comparing more than two groups. $\mathrm{P}<$ 0.05 was considered to indicate statistical significance.

\section{Results}

\section{MEG3 was increased during osteogenic differentiation of BMSCs.}

To determine whether Meg3 involved in osteogenic differentiation of BMSCs, we examined the expression Meg3 in BMSCs at day 0, 7, and 14 after osteoblastic induction. Compared with undifferentiated BMSCs, the expression of Meg3 was significantly increased at day 7 and 14 during the process of osteogenic differentiation $(P<0.05$, Figure $1 A)$.

To understand the role of Meg3 during osteogenic differentiation, we first performed Meg3 knockdown in the BMSCs using a lentivirus vector. Immunofluorescence showed that the RFP marker was stably expressed in the BMSCs (Figure 1B). PCR results showed the expression of Meg3 in the lenti-Meg3 treated BMSCs (sh-Meg3 group) were knockdown (0.7-fold) when compared with the mock treated BMSCs (blank group) and lenti-control treated BMSCs (sh-Ctrl group) $(P<0.05$, Figure $1 C)$. CCK8 results showed that no significant difference was detected in the cell proliferation rate between sh-Meg3 group and sh-Ctrl group at day 1,3, and 7, which indicate Meg3 knockdown did not affect BMSCs proliferation (Figure supplement).

\section{MEG3 knockdown promotes osteogenic differentiation of BMSCs in vitro.}

To further explore the function of Meg3 knockdown during osteogenic differentiation, the levels of osteospecific genes, including ALP, RUNX2, and osteocalcin (OCN) were detected by qPCR. qPCR analysis revealed that ALP, RUNX2, and OCN mRNA levels were significantly higher in sh-Meg3 group at day 7, and 14 than in blank group and sh-Ctrl group $(P<0.05$, Figure $2 A-C)$.

We evaluated ALP activity, an early marker of osteogenesis, at day 14 during osteogenic differentiation. Compared with the blank group and sh-Ctrl group, higher ALP activity was observed in sh-Meg3 group ( $P$ $<0.05$, Figure 2D and 2F). Calcium deposits were also examined by ARS, and the staining areas were quantified by measuring the absorbance at $560 \mathrm{~nm}$. More calcium deposits appeared in the sh-Meg3 group than in blank group and sh-Ctrl group at day $28(P<0.05$, Figure $2 \mathrm{D}$ and $2 \mathrm{E})$.

\section{MEG3 knockdown activates Wnt/ $\beta$-catenin signaling pathway in BMSCs.}

Wnt/ $\beta$-catenin signaling pathway plays an important role in the osteogenic differentiation of BMSCs. To gain insights into the mechanism by which Meg3 regulates osteogenic differentiation of BMSCs, the 
expression changes of $\beta$-catenin in BMSCs were performed. Western blot results showed that the protein level of $\beta$-catenin increased in the sh-Meg3 group when compared with sh-Ctrl group $(P<0.05$, Figure $3 A$ and $3 \mathrm{~B})$. DKK1 was previously reported as an inhibitor of Wnt/ $\beta$-catenin pathway.

To verify the relevance of the Wnt/ $\beta$-catenin pathway and Meg3 knockdown, we evaluated the expression of $\beta$-catenin between sh-Ctrl group and sh-Meg3 group treated with or without DKK1. Western blot results showed that Meg3 knockdown resulted in an increase of $\beta$-catenin in sh-Meg3 group compared to the shCtrl group, and the upregulation of $\beta$-catenin could inhibit by DKK1 $(P<0.05$, Figure $3 C$ and 3D). Taken together, these data indicate that Meg3 activates the Wnt/ $\beta$-catenin signaling pathway in BMSCs.

\section{Wnt/ $\beta$-catenin inhibition impairs MEG3-induced osteogenic differentiation of BMSCs.}

Next, we determined whether the osteogenesis effect of Meg3 knockdown on BMSCs was mediated via the Wnt/ $\beta$-catenin pathway. After treated with or without DKK1, osteo-specific genes of OCN and Runx2 were detected by qPCR in sh-Ctrl group and sh-Meg3 group. Compared with the sh-Ctrl group, silencing of Meg3 increased the expression of OCN and Runx2 in sh-Meg3 group, whereas the increasing of expression of OCN and Runx2 could blocked by DKK1 in sh-Meg3 group $(P<0.05$, Figure 4A and 4B).

Moreover, there was also less matrix mineralization at day 28 of osteogenic differentiation in the shMEG3 group treated with DKK1than in sh-MEG3 group (Figure 4C and 4D). Blocking the Wnt/ $\beta$-catenin pathway was able to reverse the osteogenesis activity induced by knockdown of Meg3 as indicated by ALP activity $(P<0.05$, Figure $4 \mathrm{E})$. These results indicate that the $W n t / \beta$-catenin pathway could mediate the osteogenesis effect of MEG3.

\section{MEG3 knockdown accelerated bone repairing in a rat critical-sized skull defect.}

To verify the pro-osteogenesis effect of Meg3 in vivo, PHMG adherent with Meg3 knockdown BMSCs were implanted in a critical-sized skull defects of rat model. The 3D morphology and 2D slice images of the newly-formed calvarial bones of each group at week 8 are reconstructed by micro-CT (Figure 5A-5C). As showed in the sagittal view, little bone growth was observed in the defect in the PHMG group (Figure 5A3) $₫$ the PHMG + sh-Ctrl group showed increased new bone formation than PHMG group (Figure 5B3). Compared with the PHMG group and the PHMG + sh-Ctrl group, newly-formed bone was apparently augmented in the PHMG + sh-Meg3 group, as the defect was almost completely filled with new calvarium, and the interfaces between the scaffold and bone tissues were connected (Figure 5C3). The local BMDs were markedly the highest at $0.497 \pm 0.043 \mathrm{~g} / \mathrm{cm}^{3}$, and there was a significant difference between the PHMG group and PHMG + sh-Meg3 groups $(P<0.05$, Figure $5 D)$. Moreover, BV/TV showed the same tendency as the BMD levels, there was a significant difference in the PHMG + sh-Meg3 group compared with the PHMG and PHMG + sh-Ctrl groups $(P<0.05$, Figure $5 E)$. These results indicate that PHMG scaffold with Meg3 knockdown BMSCs can synergistically improve bone regeneration compared in vivo. 
HE staining clearly showed that barely any new bone formation was found in the PHMG group (Figure 6A1). Only a small amount of new bone formation was observed in the PHMG + sh-Ctrl group (Figure 6A2). While in the PHMG + sh-Meg3 group, the ingrowth of new bone formation was evident in the central area of the defects as well as in the peripheral area near the pre-existing bones (Figure 6A3). The osteogenic marker $\mathrm{OCN}$ was also detected by $\mathrm{IHC}$ staining of decalcified craniums of each group. The results showed that there was less positive staining for OCN in the PHMG group (Figure 6B1). Positive brown staining for OCN was more apparent in the PHMG + sh-Ctrl (Figure 6B2) and PHMG + sh-Meg3 groups (Figure 6B3). HE and OCN IHC staining analysis of bone regeneration in calvarial defects indicated that Meg3 knockdown in BMSCs can increase bone regeneration.

\section{Discussion}

In this study, we found the expression of MEG3 was upregulated during osteogenesis in BMSCs. Furthermore, downregulation of MEG3 could promote the osteogenic differentiation of BMSCs. Moreover, we showed that decreasing the expression of MEG3 in BMSCs could markedly accelerate bone repairing with upregulated bone mineral density, bone volume, and increased new bone generation in rat criticalsized skull defects model. Mechanistically, we found that silencing of MEG3 could promoted osteogenic differentiation of BMSCs via the Wnt/ $\beta$-catenin signaling pathway. To our knowledge, this is the first report demonstrating that MEG3 enhances osteogenic differentiation of BMSCs, at least partly through activation of the Wnt/ $\beta$-catenin signaling pathway.

BMSCs combined with biomedical materials, hold great promise for regenerative medicine, especially for treating the unmet critical-sized bone defects in clinics. Therefore, effectively enhancing BMSCs osteogenic differentiation and BMSCs-mediated bone regeneration are crucial in bone tissue regeneration. Recent evidences have revealed that dysregulation of MEG3 is closely associated with bone or bone degenerative diseases. For example Liu et al. found MEG3 is up-regulated in non-union fracture bone(16) while MEG3 is downregulated in Osteoarthritis $(26,27)$. In this study, we found the expression of MEG3 was upregulated during osteogenesis in BMSCs. RUNX2 is a master transcription factor involved in osteogenic differentiation. The expression of RUNX2 was significantly increased following the downregulation of MEG3. The levels of an early marker of osteogenic differentiation (ALP) and late markers of osteogenic differentiation (OCN) were also increased due to MEG3 depression. In agreement with our results, Li et al. also found the expression of serum IncRNA MEG3 was increased in fracture patients and intervention with MEG3 siRNA could obviously promote the proliferation and differentiation of osteoblast cell line MC3T3-E1 in vitro(24). In addition, better bone healing was also observed when MEG3-modified BMSCs scaffold with PHMG used in a rat critical-sized skull defects model. Similarly, Liu et al. showed that silencing MEG3 could accelerate tibia fraction healing in mice(16). However, some other studies have revealed the contradictory roles of MEG3 in osteogenic differentiation of BMSCs. Chen et al. found MEG3 may increase osteogenic differentiation of BMSCs in osteoporosis(28), and Zhuang et al. showed overexpression of MEG3 enhanced osteogenic differentiation of BMSCs from multiple myeloma patients(15). Zheng et al. found MEG3 involved in the loss of odontogenic potential in dental mesenchymal cells. Since MEG3 was significantly downregulated in cultured dental mesenchymal cells 
but were upregulated in odontogenic dental mesenchymal tissues(29). Therefore, different expression of MEG3 in different cell types, tissues or diseases indicate MEG3 may have specifically function under certain states.

Wnt/ $\beta$-catenin signaling is an essential pathway in the osteogenic differentiation of MSCs and bone maintenance. Wnt signaling results in cellular accumulation of Wnt/ $\beta$-catenin, followed by nuclear translocation of $\beta$-catenin and activation of target genes $(17,30)$. A previous study reported crosstalk between MEG3 and Wnt/ $\beta$-catenin signaling in stem cells; that is, down-regulated MEG3 promotes osteogenic differentiation of human dental follicle stem cells by epigenetically regulating Wnt pathway(13). Gong et al. also found highly expressed MEG3 could weaken Wnt/ $\beta$-catenin signaling in glioma(22). MEG3 was reported located in the cytoplasm(13) and nucleus(16). In this study, higher $\beta$ catenin accumulation was observed following downregulation of MEG3 during osteogenesis, suggesting that MEG3 downregulation activates $\beta$-catenin-mediated transcription. Furthermore, the increased osteogenesis of BMSCs by MEG3 downregulation was blocked by an inhibitor of Wnt/ $\beta$-catenin. Since after the inhibition of canonical Wnt signaling pathway by DKK1, the osteogenic differentiation marker ALP, OCN and Runx2 were downregulated and less mineralized nodules were formed. Together, our data suggested that downregulated MEG3 might promote osteogenesis of BMSCs by partly activating the Wnt/ $\beta$-catenin signaling pathway.

The present study has some limitations. First, although we indicated that down-regulation of MEG3 mediates the Wnt/ $\beta$-catenin signaling pathway to promote osteogenic differentiation of BMSCs, it is probably involved in the activation of other signaling pathways as well. Second, the mechanisms of MEG3 interact with Wnt/ $\beta$-catenin has not been clarified completely, and thus further studies are needed.

\section{Conclusion}

Based on our data, we found that downregulation of MEG3 could promote osteogenic differentiation of BMSCs and bone repairing, partly through activation of the Wnt/ $\beta$-catenin signaling pathway. MEG3modified BMSCs can be used as a novel promising strategy for skull defects.

\section{Abbreviations}

IncRNA: long non-coding RNA; MEG3: maternally expressed gene 3; BMSCs: bone marrow mesenchymal stem cells; PHMG: poly (3-hydroxybutyrate-co-3-hydroxyhexanoate, $\mathrm{PHBHHx}$ )-mesoporous bioactive glass; DKK1: dickkopf-1; ALP: alkaline phosphatase; ARS: Alizarin red staining; OCN: Osteocalcin; Runx2: Runt-related transcription factor 2; CCK-8: Cell Counting Kit-8; mCT: Micro-computed tomography; IHC: Immunohistochemical; HE: hematoxylin and eosin; BMD: bone mineral density; BV: bone volume; TV: total volume; qPCR: quantitative reverse-transcriptase polymerase chain reaction.

\section{Declarations}




\section{Funding}

This study was supported by the National Nature Science Foundation of China (Grant No. 81801214 , 81802154).

\section{Authors' contributions}

YCQ and LMQ conceived the study, designed the experiments, reviewed and edited the manuscript. LJ and QX performed the experiments of BMSCs and wrote the manuscript; MHS and ZSH were responsible for animal handling and surgery. GGY and XZC performed statistical analysis of all experimental data. All authors read and approved the final manuscript.

\section{Acknowledgments}

The authors thank Zhang Wei, Department of Orthopedics, Second Affiliated Hospital, School of Medicine, Zhejiang University, for providing DKK1 in some in vitro experiments.

\section{Declarations}

\section{Ethics approval and consent to participate}

All methods were carried out in accordance with relevant guidelines and regulations of the Research Ethics Committee of the Shanghai General Hospital.

\section{Consent for publication}

Not applicable.

\section{Availability of data and materials}

The datasets generated/analyzed during the current study are available.

\section{Competing interests}

The authors declared that they have no competing interests.

\section{References}

1. Yeap MC, Tu PH, Liu ZH, Hsieh PC, Liu YT, Lee CY, et al. Long-Term Complications of Cranioplasty Using Stored Autologous Bone Graft, Three-Dimensional Polymethyl Methacrylate, or Titanium Mesh After Decompressive Craniectomy: A Single-Center Experience After 596 Procedures. World neurosurgery. 2019 Aug;128:e841-e50. PubMed PMID: 31082551.

2. Giese $\mathrm{H}$, Meyer J, Unterberg A, Beynon C. Long-term complications and implant survival rates after cranioplastic surgery: a single-center study of 392 patients. Neurosurgical review. 2020 Aug 26. PubMed PMID: 32844249. 
3. Cabbad NC, Stalder MW, Arroyave A, Wolfe EM, Wolfe SA. Autogenous Bone Cranioplasty: Review of a 42-Year Experience by a Single Surgeon. Plastic and reconstructive surgery. 2019 Jun;143(6):171323. PubMed PMID: 31136489.

4. Liu X, Bao C, Xu HHK, Pan J, Hu J, Wang P, et al. Osteoprotegerin gene-modified BMSCs with hydroxyapatite scaffold for treating critical-sized mandibular defects in ovariectomized osteoporotic rats. Acta biomaterialia. 2016 Sep 15;42:378-88. PubMed PMID: 27318268.

5. Qi X, Liu Y, Ding ZY, Cao JQ, Huang JH, Zhang JY, et al. Synergistic effects of dimethyloxallyl glycine and recombinant human bone morphogenetic protein-2 on repair of critical-sized bone defects in rats. Scientific reports. 2017 Feb 23;7:42820. PubMed PMID: 28230059. Pubmed Central PMCID: 5322391.

6. Qi X, Huang Y, Han D, Zhang J, Cao J, Jin X, et al. Three-dimensional poly (epsiloncaprolactone)/hydroxyapatite/collagen scaffolds incorporating bone marrow mesenchymal stem cells for the repair of bone defects. Biomedical materials. 2016 Mar 10;11(2):025005. PubMed PMID: 26964015.

7. Mondal T, Subhash S, Vaid R, Enroth S, Uday S, Reinius B, et al. MEG3 long noncoding RNA regulates the TGF-beta pathway genes through formation of RNA-DNA triplex structures. Nat Commun. 2015;6:7743. PubMed PMID: 26205790. Pubmed Central PMCID: 4525211.

8. Gordon FE, Nutt CL, Cheunsuchon P, Nakayama Y, Provencher KA, Rice KA, et al. Increased expression of angiogenic genes in the brains of mouse meg3-null embryos. Endocrinology. 2010 Jun;151(6):2443-52. PubMed PMID: 20392836. Pubmed Central PMCID: 2875815.

9. Liu J, Li Q, Zhang KS, Hu B, Niu X, Zhou SM, et al. Downregulation of the Long Non-Coding RNA Meg3 Promotes Angiogenesis After Ischemic Brain Injury by Activating Notch Signaling. Molecular neurobiology. 2017 Dec;54(10):8179-90. PubMed PMID: 27900677. Pubmed Central PMCID: 5684256.

10. Liu Y, Zeng X, Miao J, Liu C, Wei F, Liu D, et al. Upregulation of long noncoding RNA MEG3 inhibits the osteogenic differentiation of periodontal ligament cells. Journal of cellular physiology. 2019 Apr;234(4):4617-26. PubMed PMID: 30256394.

11. Zhao LD, Xu WC, Cui J, Liang YC, Cheng WQ, Xin BC, et al. Long non-coding RNA maternally expressed gene 3 inhibits osteogenic differentiation of human dental pulp stem cells via microRNA543 /smad ubiquitin regulatory factor 1/runt-related transcription factor 2 axis. Archives of oral biology. 2020 Jul 9;118:104838. PubMed PMID: 32711339.

12. Wang Q, Li Y, Zhang Y, Ma L, Lin L, Meng J, et al. LncRNA MEG3 inhibited osteogenic differentiation of bone marrow mesenchymal stem cells from postmenopausal osteoporosis by targeting miR-133a3p. Biomedicine \& pharmacotherapy = Biomedecine \& pharmacotherapie. 2017 May;89:1178-86. PubMed PMID: 28320084.

13. Deng L, Hong H, Zhang $X$, Chen D, Chen Z, Ling J, et al. Down-regulated IncRNA MEG3 promotes osteogenic differentiation of human dental follicle stem cells by epigenetically regulating Wnt 
pathway. Biochemical and biophysical research communications. 2018 Sep 10;503(3):2061-7. PubMed PMID: 30103943.

14. Li Z, Jin C, Chen S, Zheng Y, Huang Y, Jia L, et al. Long non-coding RNA MEG3 inhibits adipogenesis and promotes osteogenesis of human adipose-derived mesenchymal stem cells via miR-140-5p. Molecular and cellular biochemistry. 2017 Sep;433(1-2):51-60. PubMed PMID: 28382492.

15. Zhuang W, Ge X, Yang S, Huang M, Zhuang W, Chen P, et al. Upregulation of IncRNA MEG3 Promotes Osteogenic Differentiation of Mesenchymal Stem Cells From Multiple Myeloma Patients By Targeting BMP4 Transcription. Stem cells. 2015 Jun;33(6):1985-97. PubMed PMID: 25753650.

16. Liu YB, Lin LP, Zou R, Zhao QH, Lin FQ. Silencing long non-coding RNA MEG3 accelerates tibia fraction healing by regulating the Wnt/beta-catenin signalling pathway. Journal of cellular and molecular medicine. 2019 Jun;23(6):3855-66. PubMed PMID: 30955246. Pubmed Central PMCID: 6533481.

17. Zhang R, Oyajobi BO, Harris SE, Chen D, Tsao C, Deng HW, et al. Wnt/beta-catenin signaling activates bone morphogenetic protein 2 expression in osteoblasts. Bone. 2013 Jan;52(1):145-56. PubMed PMID: 23032104. Pubmed Central PMCID: 3712130.

18. Ahmadzadeh A, Norozi F, Shahrabi S, Shahjahani M, Saki N. Wnt/beta-catenin signaling in bone marrow niche. Cell and tissue research. 2016 Feb;363(2):321-35. PubMed PMID: 26475718.

19. MacDonald BT, He X. Frizzled and LRP5/6 receptors for Wnt/beta-catenin signaling. Cold Spring Harbor perspectives in biology. 2012 Dec 1;4(12). PubMed PMID: 23209147. Pubmed Central PMCID: 3504444.

20. Zhang W, Xue D, Yin H, Wang S, Li C, Chen E, et al. Overexpression of HSPA1A enhances the osteogenic differentiation of bone marrow mesenchymal stem cells via activation of the Wnt/betacatenin signaling pathway. Scientific reports. 2016;6:27622. PubMed PMID: 27279016. Pubmed Central PMCID: 4899801.

21. Song L, Liu M, Ono N, Bringhurst FR, Kronenberg HM, Guo J. Loss of wnt/beta-catenin signaling causes cell fate shift of preosteoblasts from osteoblasts to adipocytes. Journal of bone and mineral research : the official journal of the American Society for Bone and Mineral Research. 2012 Nov;27(11):2344-58. PubMed PMID: 22729939. Pubmed Central PMCID: 3474875.

22. Gong X, Huang M. Long non-coding RNA MEG3 promotes the proliferation of glioma cells through targeting Wnt/beta-catenin signal pathway. Cancer gene therapy. 2017 Sep;24(9):381-5. PubMed PMID: 29027534.

23. Li L, Pei S, Sun N. MEG3 targets miR-184 and Wnt/beta-catenin and modulates properties of osteosarcoma. Frontiers in bioscience. 2020 Jun 1;25:1901-12. PubMed PMID: 32472764.

24. Li XG, Liu SC, Qiao XF, Kong Y, Liu JG, Peng XM, et al. LncRNA MEG3 promotes proliferation and differentiation of osteoblasts through Wnt/beta-catenin signaling pathway. European review for medical and pharmacological sciences. 2019 Jun;23(11):4521-9. PubMed PMID: 31210279.

25. Yun HM, Park KR, Quang TH, Oh H, Hong JT, Kim YC, et al. 2,4,5-Trimethoxyldalbergiquinol promotes osteoblastic differentiation and mineralization via the BMP and Wnt/beta-catenin pathway. Cell 
death \& disease. 2015 Jul 16;6:e1819. PubMed PMID: 26181200. Pubmed Central PMCID: 4650732.

26. Su W, Xie W, Shang Q, Su B. The Long Noncoding RNA MEG3 Is Downregulated and Inversely Associated with VEGF Levels in Osteoarthritis. BioMed research international. 2015;2015:356893. PubMed PMID: 26090403. Pubmed Central PMCID: 4454735.

27. Chen K, Zhu H, Zheng MQ, Dong QR. LncRNA MEG3 Inhibits the Degradation of the Extracellular Matrix of Chondrocytes in Osteoarthritis via Targeting miR-93/TGFBR2 Axis. Cartilage. 2019 Jun 28:1947603519855759. PubMed PMID: 31253047.

28. Chen S, Jia L, Zhang S, Zheng Y, Zhou Y. DEPTOR regulates osteogenic differentiation via inhibiting MEG3-mediated activation of BMP4 signaling and is involved in osteoporosis. Stem cell research \& therapy. 2018 Jul 4;9(1):185. PubMed PMID: 29973283. Pubmed Central PMCID: 6033203.

29. Zheng Y, Jia L. Long noncoding RNAs related to the odontogenic potential of dental mesenchymal cells in mice. Archives of oral biology. 2016 Jul;67:1-8. PubMed PMID: 26986487.

30. Rudnicki MA, Williams BO. Wnt signaling in bone and muscle. Bone. 2015 Nov;80:60-6. PubMed PMID: 26453496. Pubmed Central PMCID: 4600531.

\section{Table}

Table 1. Quantitative real-time polymerase chain reaction primer sequences

\begin{tabular}{lcc}
\hline Gene & Direction & Primer sequence $\left(5^{\prime}-3^{\prime}\right)$ \\
\hline human GAPDH & Forward & ATCCCATCACCATCTTCC \\
& Reverse & GAGTCCTTCCACGATACCA \\
human ALP & Forward & GTTTTCTGTTCTGTAAGACGGG \\
& Reverse & GCCGTTAATTGACGTTCCGA \\
human RUNX-2 & Forward & CCGAGCTACGAAATGCCTCT \\
& Reverse & GGACCGTCCACTGTCACTTT \\
human OCN & Forward & CCCCCTCTAGCCTAGGACC \\
& Reverse & ACCAGGTAATGCCAGTTTGC \\
human MEG3 & Forward & GTTGAGCCTTCAGTGTCTGCAT \\
& Reverse & GCTTTGGAACCGCATCACA \\
\hline
\end{tabular}

\section{Figures}


A

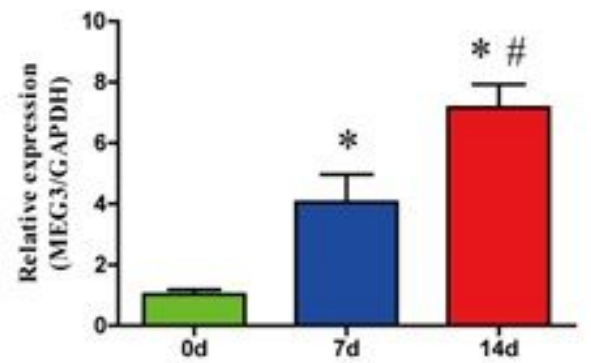

B

light microscope

RFP

sh-Ctrl
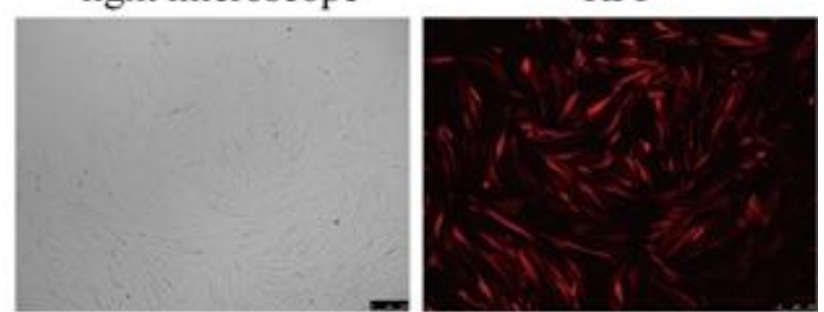

sh-Meg3
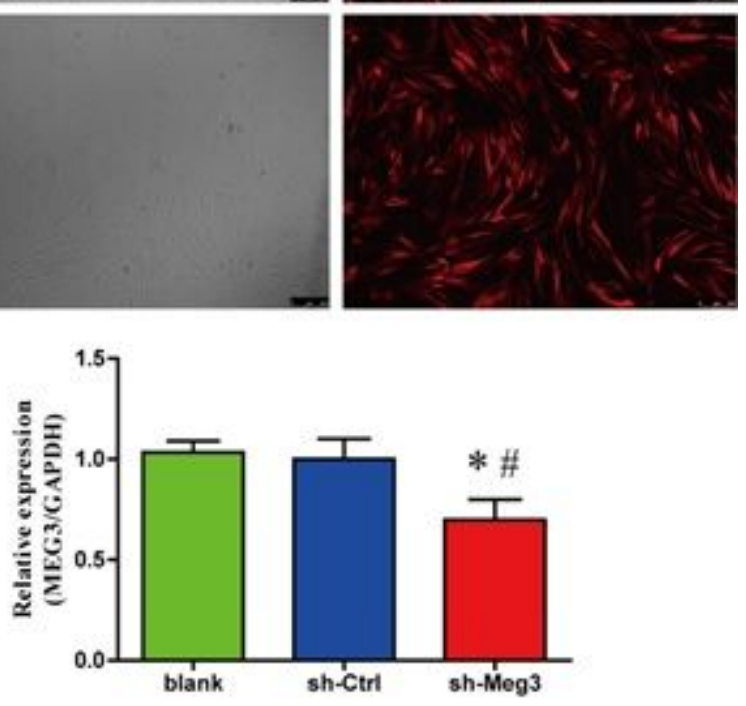

\section{Figure 1}

Expression of Meg3 during BMSCs osteogenic differentiation and construction of Meg3 downregulated BMSCs. (A) Bar graphs show the expression levels of Meg3 in BMSCs at days 0,7 and 14 of osteogenic differentiation. (B) BMSCs after lentiviral transfection were observed under a normal microscope and a fluorescence microscope. (C) The expression of Meg3 was determined by qPCR in lentivirus blank group, sh-Ctrl group and sh-Meg3 group ( $n=3$ /group). * $P<0.05$ versus the blank group. \#P< 0.05 versus shCtrl group. 

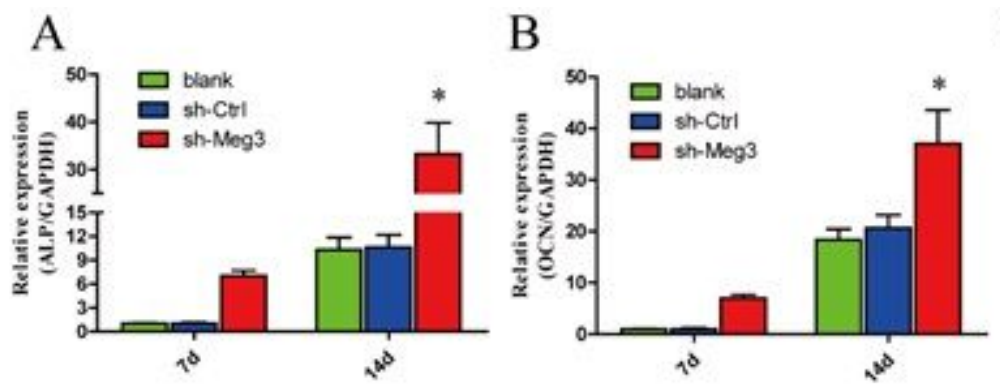

$\mathrm{C}$

$\mathrm{D}$

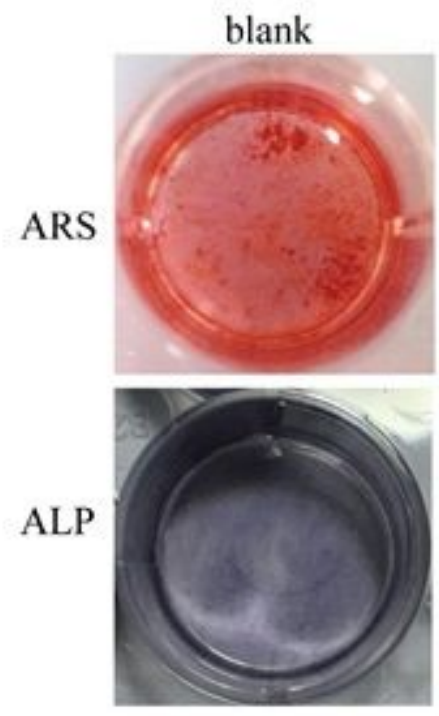

sh-Ctrl
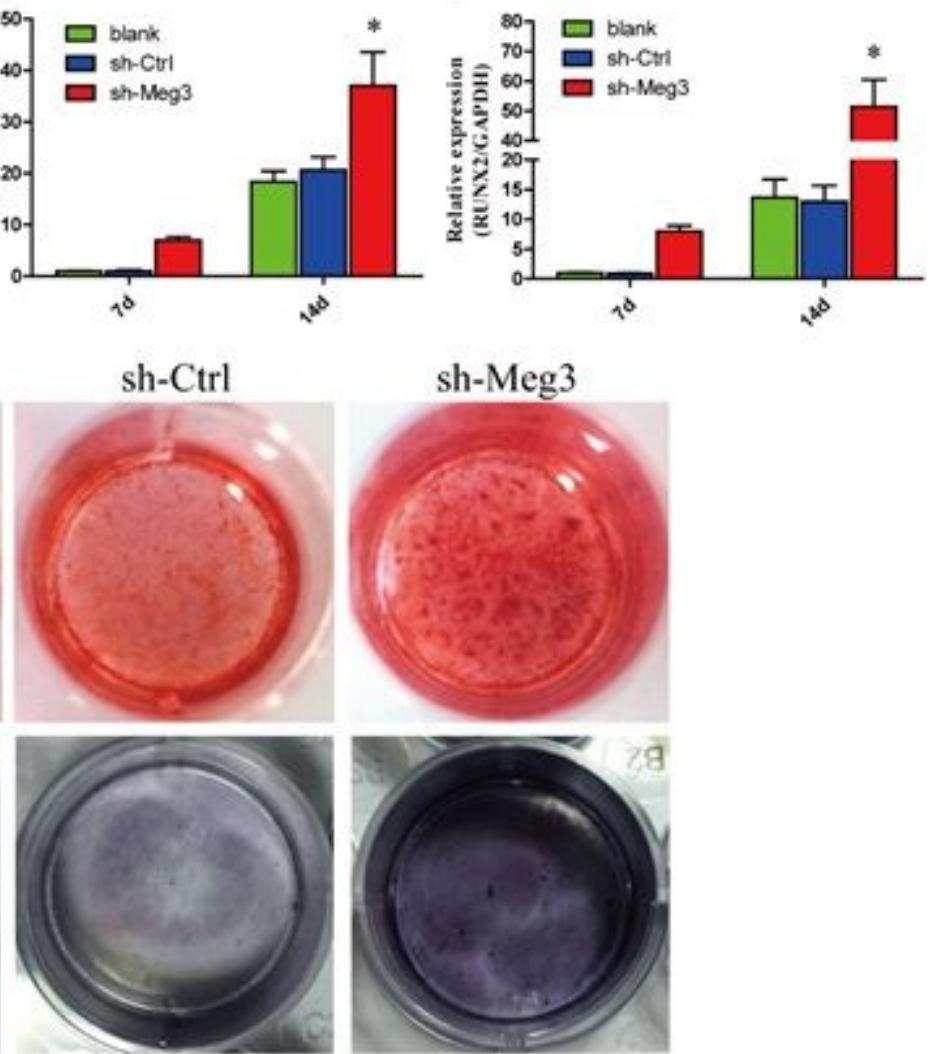

sh-Meg3

$\mathrm{E}$

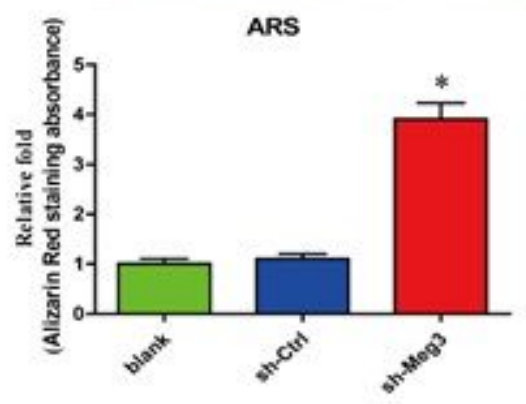

F $\left.\quad{ }^{2.5}\right] \quad$ ALP
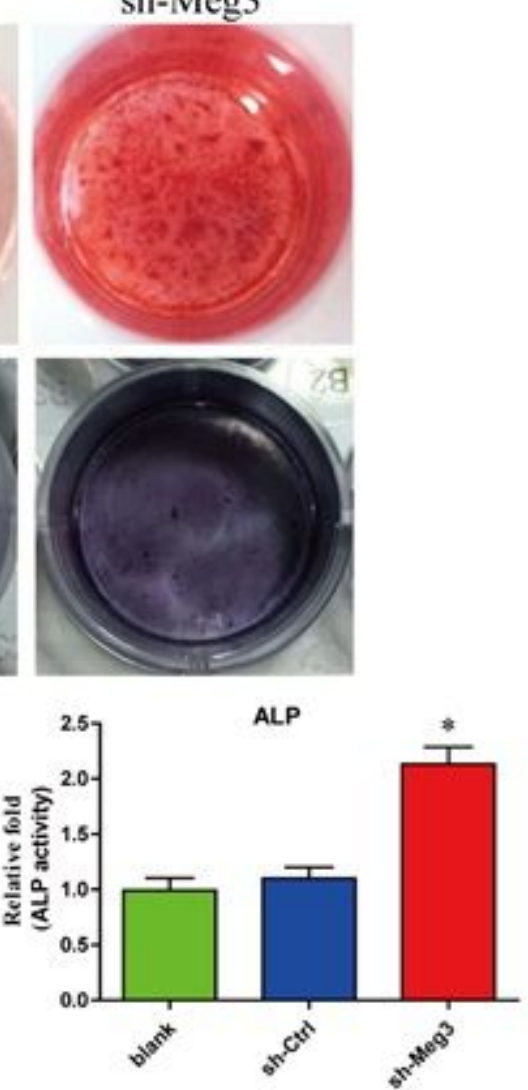

Figure 2

Meg3 knockdown promoted osteogenic differentiation of BMSCs. (A) Bar graph shows the qRT-PCR analysis of ALP expression at days 7, and 14 of osteogenic differentiation. (B) Bar graph shows the qRTPCR analysis of OCN expression at days 7, and 14 of osteogenic differentiation. (C) Bar graph shows the qRT-PCR analysis of RUNX2 expression at days 7, and 14 of osteogenic differentiation. (D) Alizarin red and ALP staining in the blank, sh-ctrl and sh-Meg3 groups at day 14 of osteogenic differentiation. (E) Alizarin red staining area in the blank, sh-ctrl and sh-Meg3 groups determined by measuring the absorbance at $560 \mathrm{~nm}$. (F) ALP activity in the blank, sh-ctrl and sh-Meg3 groups at day 14 of osteogenic differentiation. Data are presented as mean $\pm S D$. ${ }^{P} P<0.05$ versus the blank group. $\# \mathrm{P}<0.05$ versus shCtrl group(n=3/group). 
A

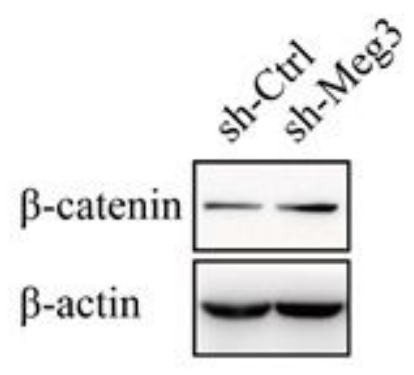

$\mathrm{C}$

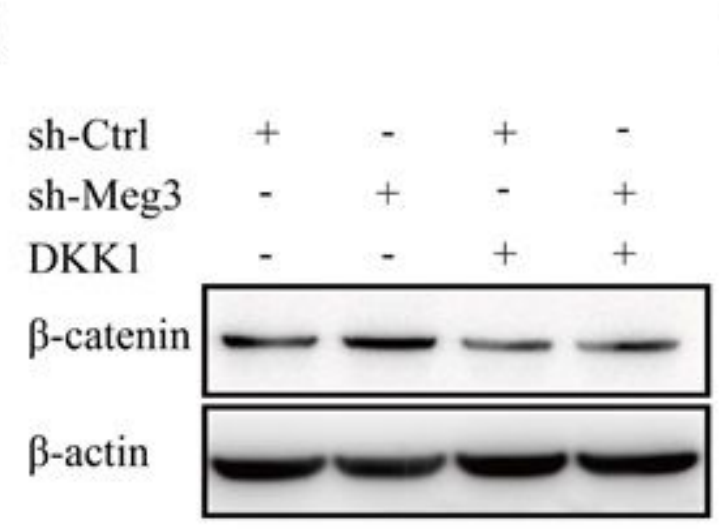

B
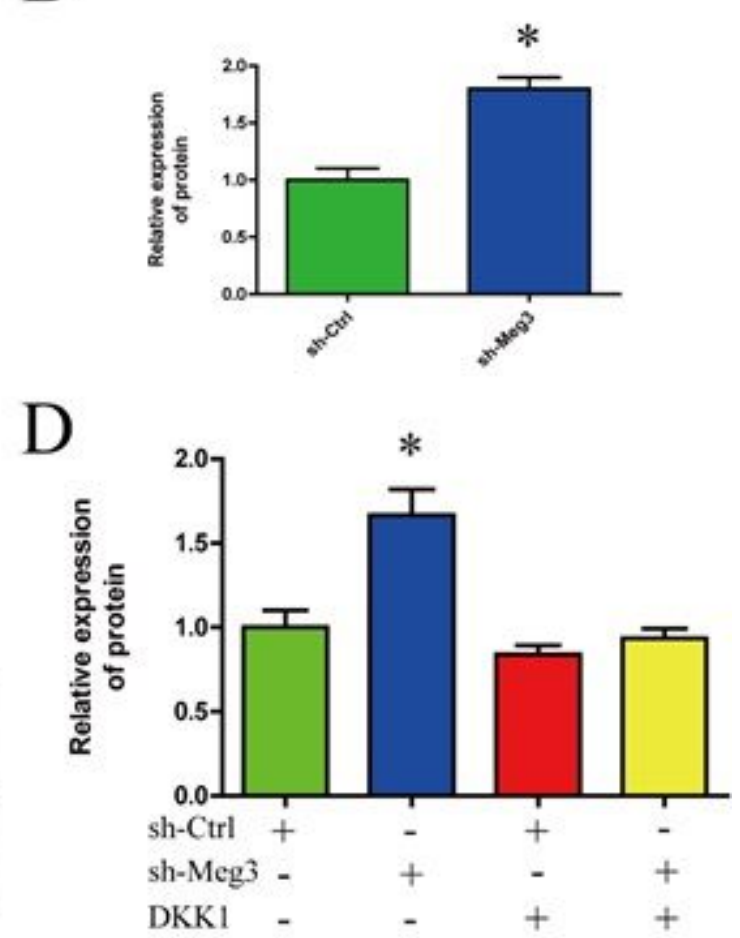

\section{Figure 3}

Meg3 regulates wnt/ $\beta$-catenin signaling. ( $A$ and $B$ ) Representative immunoblot and bar graph show the relative expression level of $\beta$-catenin in sh-ctrl and sh-Meg3. (C) sh-Ctrl and sh-Meg3 BMSCs were treated with or without Wnt/ $\beta$-catenin signaling inhibitor (DKK1). The expression of $\beta$-catenin in sh-ctrl, sh-ctrl + DKK1, sh-Meg3, and sh-Meg3 + DKK1 groups were determined by Western blot analysis. (D) Bar graph shows quantification expression of $\beta$-catenin in sh-ctrl, sh-ctrl + DKK1, sh-Meg3, and sh-Meg3 + DKK1 groups ( $n=3 /$ group). $* P<0.05$ versus sh-ctrl. 


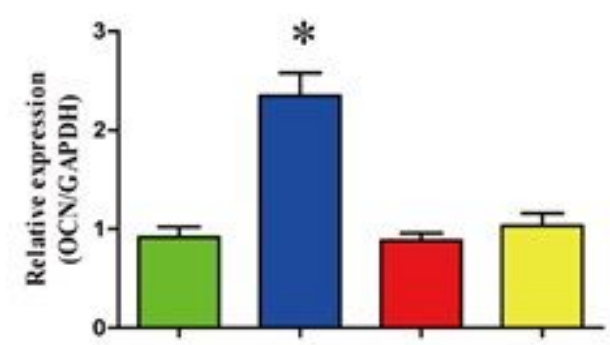

$\mathrm{B}$
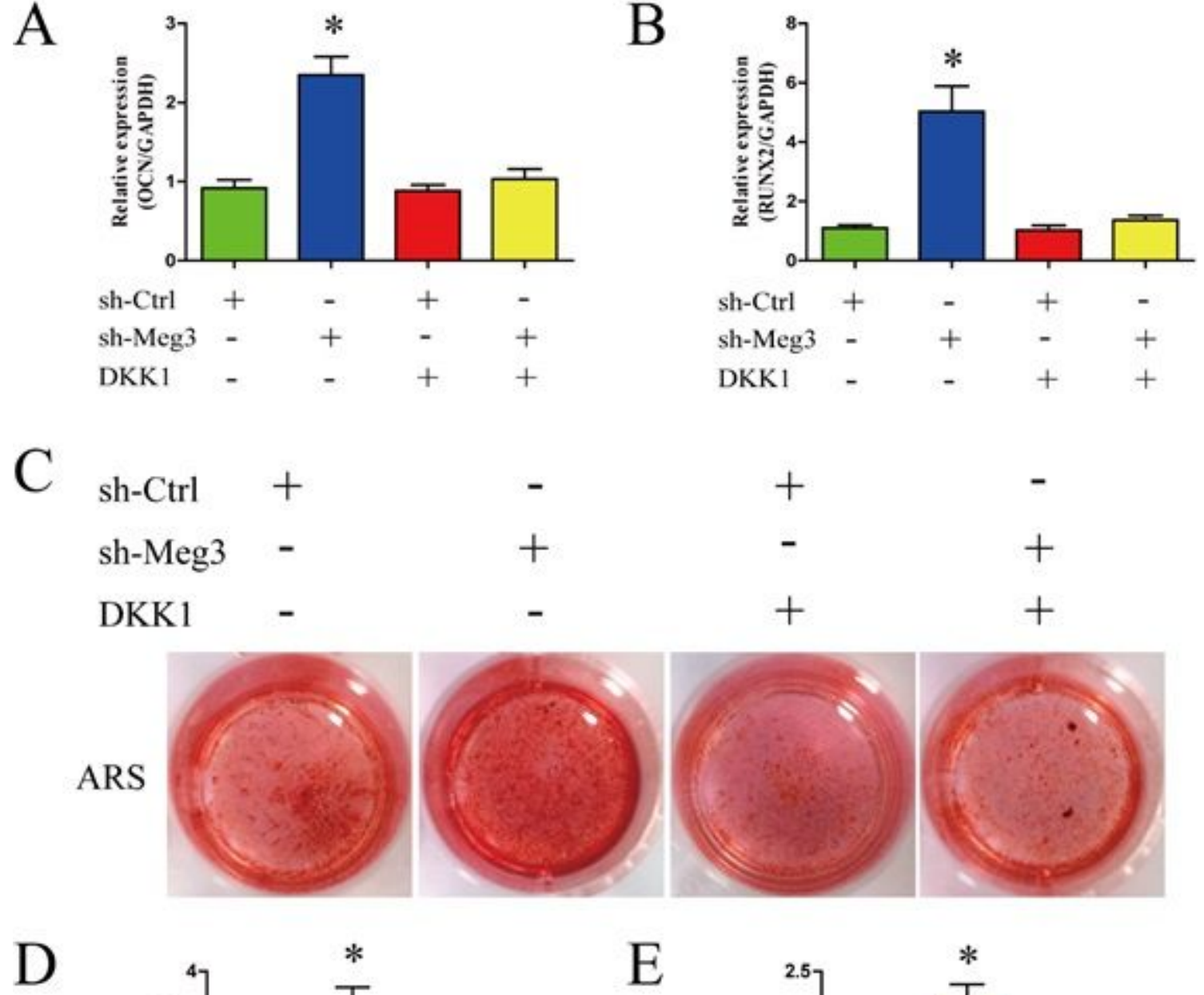

$\begin{array}{lllll}\operatorname{sh}-\mathrm{Ctrl} & + & - & + & - \\ \operatorname{sh}-\mathrm{Meg} 3 & - & + & - & + \\ \text { DKK1 } & - & - & + & +\end{array}$

$\mathrm{D}$

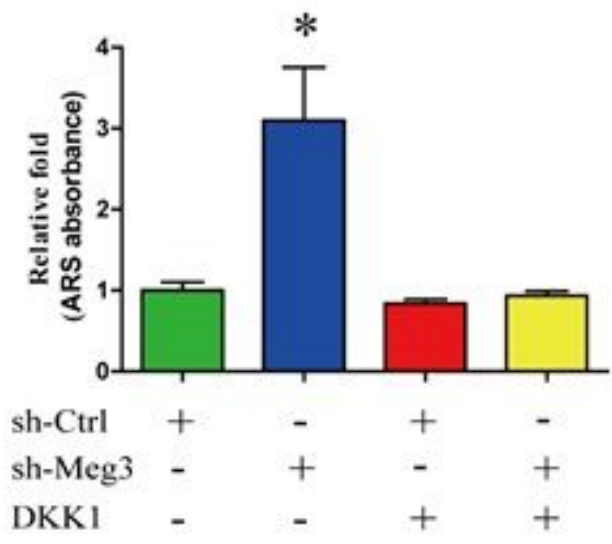

$\mathrm{E}$

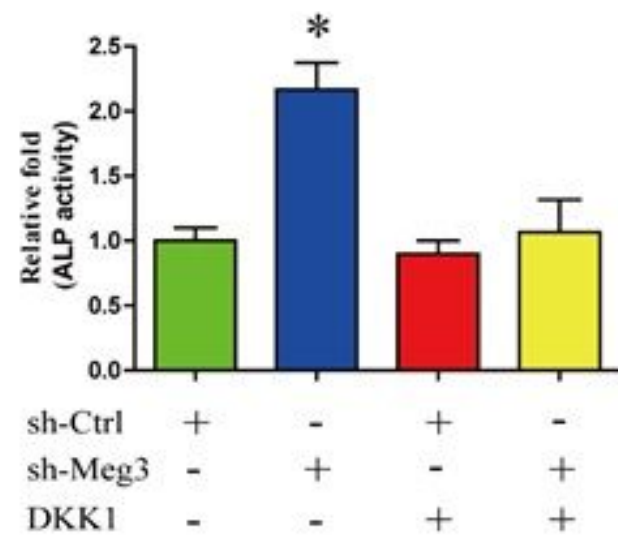

Figure 4

Wnt/ $\beta$-catenin inhibition impairs Meg3-induced osteogenesis of BMSCs. (A) sh-Ctrl and sh-Meg3 BMSCs were treated with or without Wnt/ $\beta$-catenin signaling inhibitor (DKK1). Bar graph shows the expression of OCN mRNA in sh-ctrl, sh-ctrl + DKK1, sh-Meg3, and sh-Meg3 + DKK1 groups. (B) Bar graph shows the expression of RUNX2 mRNA in sh-ctrl, sh-ctrl + DKK1, sh-Meg3, and sh-Meg3 + DKK1 groups. (C) Alizarin red staining in the sh-ctrl, sh-ctrl + DKK1, sh-Meg3, and sh-Meg3 + DKK1 groups at day 14 of osteogenic differentiation. (D) Alizarin red staining area in the sh-ctrl, sh-ctrl + DKK1, sh-Meg3, and sh-Meg3 + DKK1 groups determined by measuring the absorbance at $560 \mathrm{~nm}$. (E) Bar graph shows expression of ALP in sh-ctrl, sh-ctrl + DKK1, sh-Meg3, and sh-Meg3 + DKK1 groups. Data are presented as mean \pm SD. * $P<$ 0.05 versus the sh-ctrl group ( $n=3 /$ group). 


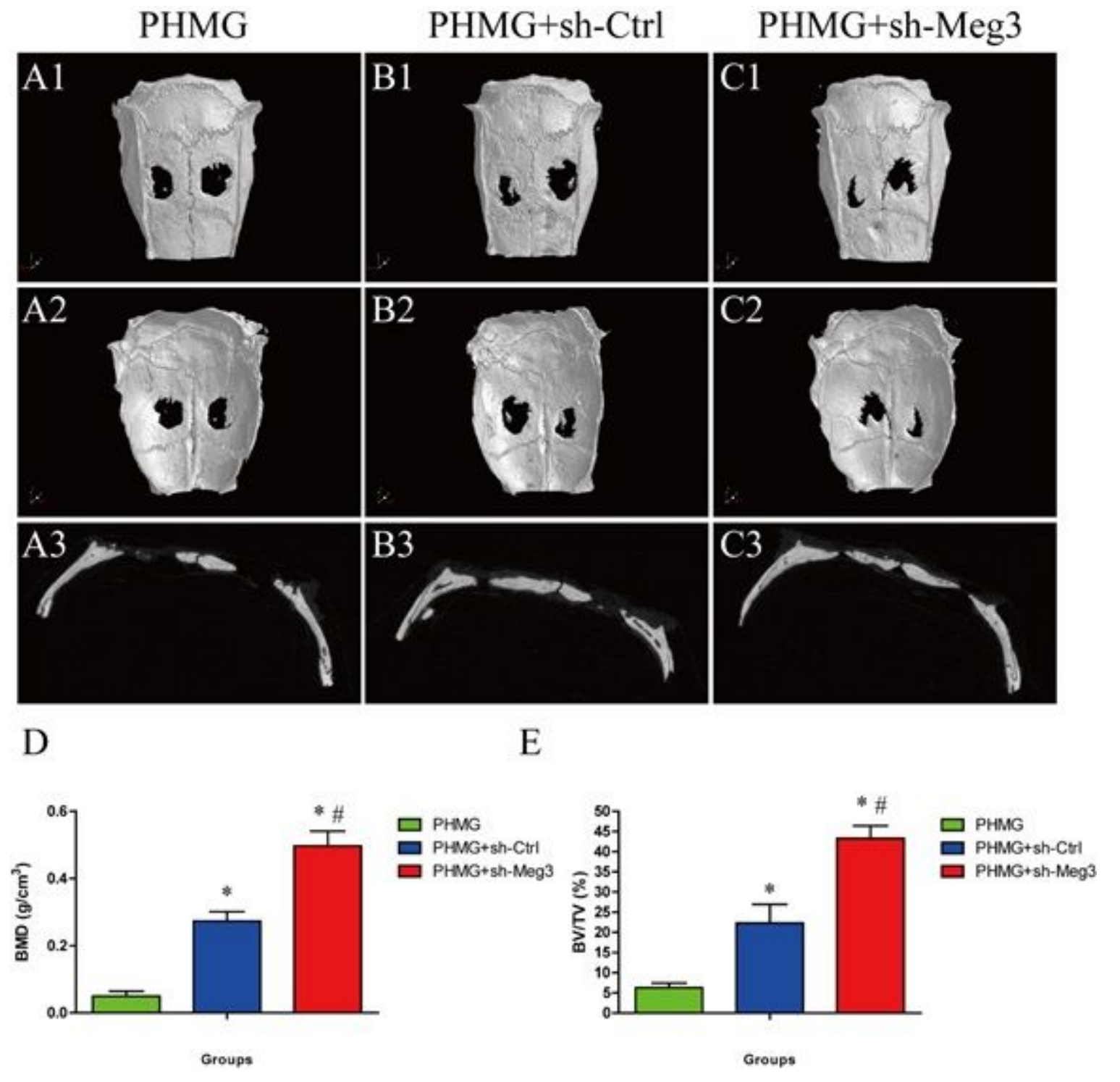

Figure 5

Meg3 knockdown in BMSCs promoted bone repairing in vivo. (A1-C3) Representative micro-CT images show the bone repairing in PHMG, PHMG + sh-Ctrl and PHMG +sh-Meg3 groups at 8 weeks. Bar graph shows quantitative analysis of micro-CT vessel volume in each group. Morphometric analysis of BMD (D) and BV/TV (E) as determined by micro-CT for each group at 8 weeks. ${ }^{\mathrm{P}}<0.05$ versus the PHMG group, $\# P<0.05$ versus $P H M G+$ sh-Ctrl group. ( $n=6 /$ group) .

\section{Supplementary Files}

This is a list of supplementary files associated with this preprint. Click to download.

- SupplementaryFigure.pdf 